\title{
Exclusion of the SCN2B gene as candidate for CMT4B
}

\author{
A lessandra Bolino ${ }^{1}, \mathrm{M}$ arco Seri ${ }^{2}$, Francesco Caroli $^{2}$, James E ubanks ${ }^{3}$, Jayashree \\ William A Catterall ${ }^{3}$ and M arcella Devoto ${ }^{1,9}$ \\ ${ }^{1} \mathrm{~L}$ aboratorio di G enetica M olecolare, Facoltà di M edicina, U niversità di $G$ enova \\ 2/ stituto G G aslini, Genova, I taly \\ ${ }^{3} \mathrm{D}$ epartment of Pharmacology, U niversity of Washington, Seattle, WA, USA \\ ${ }^{4}|\mathrm{~B}| \mathrm{G}$, Facoltà di M edicina, U niversità di G enova \\ ${ }^{5}$ D ipartimento di Scienze N eurologiche e della Riabilitazione, U niversità di G enova \\ 6 Istituto di N eurologia, Facoltà di M edicina, U niversità di Reggio Calabria, Catanzaro \\ ${ }^{7}$ I stituto di M edicina Sperimentale e B iotecnologia, CNR, Cosenza, I taly \\ ${ }^{8}$ International A gency for Research on Cancer, Lyon, France \\ ${ }^{9} \mathrm{~L}$ aboratory of Statistical G enetics, Rockefeller University, N ew York, NY, USA
} Srinivasan ${ }^{3}$, Paola Mandich ${ }^{4}$, A ngelo Schenone ${ }^{5}$, A Ido Q uattrone ${ }^{6,7}$, G iovanni R omeo ${ }^{1,8}$,

\begin{abstract}
Charcot-Marie-Tooth disease type 4B (CMT4B) is a demyelinating autosomal recessive motor and sensory neuropathy characterised by focally folded myelin sheaths in the peripheral nerve. The CMT4B gene has been localised by homozygosity mapping and haplotype sharing in the 11 q23 region. A cDNA encoding for the $\beta 2$ subunit of the human brain sodium channel, SCN2B, has been recently assigned to the same chromosomal interval by FISH. The SCN2B gene has been considered a good candidate for CMT4B on the basis of protein homology, chromosomal localisation, and putative biological function of the coded product. In this paper, we report the genomic structure of the SCN2B gene consisting of 4 exons and 3 introns spanning a region of approximately $12 \mathrm{~Kb}$. In addition, a search for mutations in patients affected with CMT4B as well as a refined physical localisation excludes SCN2B as the CMT4B gene.
\end{abstract}

Keywords: peripheral neuropathy; sodium channel; genomic structure; physical mapping

\section{Introduction}

A mong the heterogeneous group of the autosomal recessive demyelinating hereditary motor and sensory neuropathies (HMSN) three pathological forms are

Correspondence: Drs Marcella Devoto and A lessandra Bolino, Laboratorio di Genetica Molecolare, Facoltà di Medicina, U niversità di G enova, Istituto G. Gaslini, L argo Gaslini 5, 16148 Genova - Italy. Tel: +39 10 5636400; Fax: +39 10 3779797; E-mail: genseq@tin.it

R eceived 5 January 1998; revised 24 M arch 1998; accepted 2 A pril 1998 now recognised, one with classical onion bulbs, one with basal lamina onion bulbs or Charcot-M arie-Tooth (CMT) type $4 \mathrm{~A},{ }^{1}$ and a third characterised by focally folded myelin sheaths or Charcot-M arie-Tooth type $4 \mathrm{~B}$ (CM T4B). ${ }^{2}$

We previously reported a large inbred pedigree with recurrence of demyelinating motor and sensory neuropathy with focally folded myelin sheaths, CMT4B, inherited as an autosomal recessive trait. ${ }^{3}$ Taking advantage of the particular inbred structure of the family, we used homozygosity mapping and haplotype sharing to localise, through a genome-wide search, the 
CM T4B gene on chromosome $11 q 23$ in a 5 cM interval between D11S1332 and D 11S917. ${ }^{4}$

Recently, a cDNA encoding the $\beta 2$ subunit of the human brain sodium channel, SCN 2B, was isolated and mapped by FISH on chromosome $11 q 23 .{ }^{5}$ The voltagegated sodium channels are membrane proteins responsible for the initiation and propagation of action potentials in most excitable cells. In the rat brain, these channels are a heterotrimeric complex composed of a pore-forming $\alpha$ subunit and two smaller auxiliary subunits, $\beta 1$ and $\beta 2 .{ }^{6,7} \mathrm{H}$ uman $\beta 2$ is a 186 amino acid glycoprotein with a short intracellular $\mathrm{COOH}$-terminal domain, a single transmembrane segment, and a large extracellular domain containing a predicted immunoglobulin (I g) V-type fold with a disulfide bond and four $\mathrm{N}$-linked glycosylation sites. The human protein has $89 \%$ amino acid sequence identity and $93 \%$ similarity to the rat $\beta 2$ and, accordingly, many of the structural properties are conserved. ${ }^{5,7}$

B ased on homology between the rat $\beta 2$ and the cell adhesion molecule contactin, it has been postulated that $\beta 2$ might interact with proteins of the extracellular matrix during the myelination process of the central and the peripheral nervous systems as suggested by the presence of $\beta 2$ in rat dorsal root ganglia. ${ }^{5}$ Correspondingly, the human $\beta 2$ has the highest similarity to another cell adhesion molecule, the myelin protein zero (p0), a major component of the peripheral myelin which contains an extracellular Ig loop between two cysteine residues and $\mathrm{N}$-linked glycosylation sites. $\mathrm{M}$ utations in myelin p0 cause an autosomal dominant form of demyelinating CMT diseases, CM T type 1B. ${ }^{8}$

$\mathrm{O} n$ the basis of the protein homologies, chromosomal localisation, and the postulated role during the myelination process, human $\mathrm{SCN} 2 \mathrm{~B}$ has been considered a potential candidate for CM T4B.

In the present study, we first characterised the genomic structure of the $\mathrm{SCN} 2 \mathrm{~B}$ gene and then demonstrated that $\mathrm{SCN} 2 \mathrm{~B}$ is not the $\mathrm{CMT} 4 \mathrm{~B}$ gene. A search for mutations in the coding region of $\mathrm{SCN} 2 \mathrm{~B}$ in CM T 4B patients yielded negative results. In addition, a refined physical localisation showed that $\mathrm{SCN} 2 \mathrm{~B}$ maps approximately $20 \mathrm{cM}$ distal to the critical region for the disease.

\section{Materials and Methods}

DNA Extraction

DNA was extracted from peripheral leukocytes of the CMT4B patients using standard methods. ${ }^{9}$ DNA extraction from positive YAC clones was performed as described elsewhere. $^{10}$

\section{Genomic Structure of the SCN2B Gene and Mutation Analysis}

$P C R$ reactions on the $S C N 2 B$ gene were performed according to standard protocols $\mathrm{s}^{9}$ using $500 \mathrm{ng}$ of genomic D NA , $200 \mu \mathrm{M}$ of each dNTP, $30 \mathrm{pmol}$ of each primer, 1 or $1.5 \mathrm{~mm}$ of [M g2 + ], and $1.25 \mathrm{U}$ of Taq polymerase, in a final volume of $50 \mu \mathrm{l}$. The same PCR conditions with $1 \mathrm{~mm}$ of [ $\mathrm{Mg2}+]$ were also used to amplify the four exons of the SCN $2 B$ gene in the CMT4B patients. The following couples of primers designed on the intron-exon boundaries were used (Figure 1 and Table 1 ): UTR -5 ' and EX $1 R$ for exon 1, with an annealing temperature of $55^{\circ} \mathrm{C}$; EX2F and IR for exon 2, using an annealing temperature of $61^{\circ} \mathrm{C}$; IF and IBR at annealing temperature of $59^{\circ} \mathrm{C}$ for exon 3; and finally, IB F 2 and E B R 3 for the last exon using an annealing temperature of $59^{\circ} \mathrm{C}$.

M olecular cloning was carried out using the $\mathrm{PCR}$ II vector kit from I nvitrogen following the manufacturer's instructions. Finally, cycle sequencing was performed using a PCR-dye primer and/or terminator protocol on an A pplied Biosystem 373A Sequencer.

Physical Mapping

Physical mapping of the $\mathrm{SCN} 2 \mathrm{~B}$ gene was performed using the $\mathrm{G}$ enB ridge $4 \mathrm{R}$ adiation $\mathrm{H}$ ybrid pane ${ }^{11}$ ( $\mathrm{R}$ esearch $\mathrm{G}$ enetiCS, H untsville, A labama, USA) as well as the CEPH megaYAC library (provided by the YAC screening Centre DIBIT, Milan, Italy).

\section{Results and Discussion}

Since only the $648 \mathrm{bp}$ open reading frame (OR F) of the human SCN2B CDNA was available, we first characterised the SCN2B genomic structure. To this end, we designed two pairs of primers which were expected to amplify two overlapping fragments of an average size of $600 \mathrm{bp}$ from human genomic DNA. This hypothesis was based on the homology to the highly conserved rat gene where two introns were identified in the coding sequence of the mature $\beta 2$ protein. ${ }^{7}$

$U$ sing the first pair of primers, EAFN and EAR (Figure 1 and Table 1), located in the 5 ' portion of the CDNA, we were not able to obtain a single amplification product, probably because of the homology between this region of the gene and molecules belonging to the Ig superfamily. On the other hand, primers $E B F$ and EBR (Figure 1 and Table 1) from the $3^{\prime}$ portion of the coding sequence amplified a fragment of approximately $1.5 \mathrm{~K}$ b which was cloned in the $\mathrm{pCR}$ II vector (Invitrogen Corporation, Carlsbad, California, CA, USA). The recombinant clones were entirely sequenced and an intron of $977 \mathrm{bp}$ was identified.

In order to obtain a single amplification product containing the $5^{\prime}$ portion of the gene using $\mathrm{SCN} 2 \mathrm{~B}$ 
5' UTR

EXON 1

intron 1

EXON 2 GGCTCTGACGCCCGCCTGCCCTGCACCTTCAACT

EAFN $\rightarrow$ $<$-INTR TGGCTACCTCGCCCTGCCTTCAGCCTCACGGGGCTCAGTCTCTTTTTCTCTTTGGgtaagttagacacat $<-$ EX1R

ctgcatgctcccgagggttcagaagtgtagccgcaatgtgaagaggatgggggcaggggataggaaagaaggcattgctagcgcttccctctgtgt ccttgggaggggaggtgctggggactggaagggacgttgctggggagtgcagaaccctgggactacttagtcacagaaatgttgggtcaggggatct $\mathrm{EX} 2 \mathrm{~F} \rightarrow$ gagtgttgctgggggctgacaattgt....//....ccaggcacagagggcttgccctccccagccctttcagccagactcctcaccagcttgtggctt EAFN3 $\rightarrow$ catctgcagTGCCACCAGGACGGAGCATGGAGGTCACAGTACCTGCCACCCTCAACGTCCTCA A T $<-$ IR CTCCCTGAACT GGACTTACCAGGAGTGCAACAACTGCTCTGAGGAGATGgtgagtcctgggctgaaggc aggggcaggggaggaggtaggagcccatggcatgaagcccetggtcccaccaccggccccggcactgcgctagggecccagaggaccctgggt intron 2 gggctgcgtgctctggaggctcttgaaacaaggtgtgctgcgcatccaggtgcctcacagaggtaactgcctctcttcccaggaaaagggtggatg IF $\rightarrow$ ggaagagggggcatcctcactgtccttgtagttccttgctcatccagccaccatcctgtccettgctccaaccccagTTCCTCCAGTTCC $\mathrm{EBF} \rightarrow \quad<-$ EBFR

GCATGAAGATCATTAACCTGAAGCTGGAGCGGTTTCAAGACCGCGTGGAGTTCTCAGGGAA EXON 3 CCCCAGCAAGTACGATGTGTCGGTGATGCTGAGAAACGTGCAGCCGGAGGATGAGGGGATTT

$$
<- \text { IBR }
$$

GGTCCTCATGGAAGGtgaaggctggggtgccagctgcggggccetgacctttcccacccacctactctctgcggcatttgctcagggca

ctttggggaggccaggccctcctgaggatgggagcaaccatttgtgctgcatagaaggaatccattttcttcctctagtcctcaagaacctgggaa catgtcccagagagtcctggtaatgatacagaatataatcccagtcttaataatggggcttggtgtgatggctcacacctgtaatcacagcgctttgg gaggctgaggcaggcagatagcttgagctcaggagctcgagaccagcctgggcaacatggcaaaactccatctctacagaaaatgcaaaaattagc

tgggcatggtggcttgcacctatactcccagctacttgggaggctgaggtgagaggatcatctgagcccagcaggtagaggctgcagtgaatcatga

Figure 1 G enomic sequence of the SC N 2B gene which was submitted to G enBank under accession nos A F 049496 and A F 049497 . Primers used in PCR amplification are underlined. The sequences of these primers are shown in detail in Table 1. The complete coding sequence of the SCN2B gene with the corresponding protein sequence was also submitted to $G$ enBank under accession no. A F049498. 
ttgtgccactgcactccagcttgggcaacagagcaagaccctgtttcaaatgacaacaacagcaataataatgatggtgatgatggcagcttcattt

intron 3 attgggcacctctatgtctcagtcatagggctaagctctgtttatgcattatctcatcctcatgaaaaccttgagggaggtactattattaagcccat ttacatgttagaaaactgaggttagagaggataagttacttaaggtcacgcaattcgtaagttgtacaattaggactcaaatttgggtctgtttgatc ttaaagttcatgctcttaaatacttcatcacactagctcctctgggggcttgggtgtcctgggatttggaggtggcggcgcttcagggtcagtcactg $\mathrm{IBF} 2 \rightarrow$

gattgcttttcggctttgctaaagtcctgtctgtggaggagagtaaagtcaggatggttccacgcatgccacgggtagtggggtgatgggggaggccc ctttccagccctggtgctcagtggctctgcttcctttccagAGCCCCCTGAGCGGGACTCCACGGTGGCCGTGATTGT GGGTGCCTCCGTCGGGGGCTTCCTGGCTGTGGTCATCTTGGTGCTGATGGTGGTCAAGTGTGTGA EXON 4

GGAGAAAAAAAGAGCAGAAGCTGAGCACAGATGACCTGAAGACCGAGGAGGAGGGCAAG $<-$ EBR

ACGGACGGTGAAGGCAACCCGGATGATGGCGCCAAGT AGtgggtggccggccctgcagcctccegtgtccegtc

$$
<- \text { EBR3 }
$$

3' UTR

tcctcccctctccgccctgtacagtgaccctgcctgctcgctcttggtgtgcttcccgtgacctaggacccagggcccacctggggectcctgaac ccccgacttcgtatctcccacctgcaccaagagtgacccactctcttccatccgagaaacctgccatgctctgggacttgtgggecctggggagag gagagaaagggctccacctgccagtccctggggggaggcaggaggcacatgttgagggtcccc

Figure 1 continued

Table 1 Primers used for PCR amplification of the SCN2B gene

\begin{tabular}{|c|c|c|c|}
\hline Primer & Sequence & Strand & Position \\
\hline $\begin{array}{l}\text { UTR-5' } \\
\text { EAFN } \\
\text { INTR } \\
\text { EX IR } \\
\text { EX 2F } \\
\text { EAFN3 } \\
\text { EAFN2 } \\
\text { IR } \\
\text { IF } \\
\text { EBF } \\
\text { EBFR } \\
\text { EAR } \\
\text { IBR } \\
\text { IBF2 } \\
\text { EBR } \\
\text { EBR3 }\end{array}$ & $\begin{array}{l}\text { TTCCTCCCTTGTAGTTCTCGC } \\
\text { TCGCCCTGCCTTCAGCCTCA } \\
\text { GAGAAAAAGAGACTGAGCCC } \\
\text { CATCCTCTTCACATTGCGGC } \\
\text { GCCCTTTCAGCCAGACTCCTC } \\
\text { CCTGCCACCCTCAACGTCCTC } \\
\text { GCACCTTCAACTCCTGCTAC } \\
\text { CTGCCTTCAGCCCAGGACTC } \\
\text { CACTGTCCTTGAGTTCCTTGC } \\
\text { GAAGCTGGAGCGGTTTCAAG } \\
\text { CTTGAAACCGCTCCAGCTTC } \\
\text { TAXTTGCTGGGGTCCCTGAGAAC } \\
\text { GTAGGTGGGTGGGAAAGGTC } \\
\text { GGTTCCACGCATGCCACGGG } \\
\text { TGGCGCCATCATCCGGGTTGC } \\
\text { AGCAGGCAGGGTCACTGTAC }\end{array}$ & $\begin{array}{l}\text { FORWARD } \\
\text { FORWARD } \\
\text { REVERSE } \\
\text { REVERSE } \\
\text { FORWARD } \\
\text { FORWARD } \\
\text { FORWARD } \\
\text { REVERSE } \\
\text { FORWARD } \\
\text { FORWARD } \\
\text { REVERSE } \\
\text { REVERSE } \\
\text { REVERSE } \\
\text { FORWARD } \\
\text { REVERSE } \\
\text { REVERSE }\end{array}$ & $\begin{array}{l}\text { 5'-UTR } \\
\text { EXON } 1 \\
\text { EXON } 1 \\
\text { INTRON } 1 \\
\text { INTRON } 1 \\
\text { EXON } 2 \\
\text { EXON } 2 \\
\text { INTRON } 2 \\
\text { INTRON } 2 \\
\text { EXON } 3 \\
\text { EXON } 3 \\
\text { EXON } 3 \\
\text { INTRON } 3 \\
\text { INTRON } 3 \\
\text { EXON } 4 \\
\text { 3'-UTR }\end{array}$ \\
\hline
\end{tabular}


specific primer sequences, we extended the sequence of the CDNA to its untranslated regions. We performed a $B L A S T$ search of sequences similar to the human SCN2B coding sequence on the Expressed Sequence Tag human CDNA database (http://www.ncbi.nih.gov/ $\mathrm{dbEST} / \mathrm{index} . \mathrm{html}$. We retrieved EST clones with high homology to $\mathrm{SCN} 2 \mathrm{~B}$ and belonging to the same contig (THC 168161, TIGR database) and we partially sequenced two of them (IMAGE Consortium Clones $I D=25456$ and ID = c-0cd03) which were expected to contain the untranslated regions of $\mathrm{SCN} 2 \mathrm{~B}$. The sequence of $190 \mathrm{nt}$ from the $5^{\prime}-U \mathrm{TR}$ and $236 \mathrm{nt}$ from the $3^{\prime}$-UTR was determined and is shown in Figure 1.

Several forward primers were then designed from the $5^{\prime}$-UTR as well as the $5^{\prime}$ end of the CDNA and used in combination with various reverse primers from the $3^{\prime}$ end of the coding sequence. Only the pair of primers EA FN 2 and EBFR (Figure 1 and Table 1 ) were able to amplify a product of approximately $450 \mathrm{bp}$ which was cloned in $\mathrm{PCR}$ II. The recombinant clones were sequenced and an additional intron of $289 \mathrm{bp}$ was found.

Because a PCR product had been obtained using primers UTR-5' with INTR and EAFN3 with IR (Figure 1 and Table 1), whereas no PCR product had been obtained using these primers in different combinations, we deduced that a large intron had to be present between primers INTR and EAFN3. In order to amplify this last intron, we screened by $P C R$ the pools from a human CEPH Mega-YAC library using EAFN2/EBFR as primers and we found three positive YAC clones (665E 10, 742F 9, and 785E 12). Clone 665E 10 was used as a template in a long PCR reaction (B oehringer, M annheim Corporation, Indianapolis, IN,
USA) using primers EAFN and IR (Figure 1 and Table 1). A PCR product of more than $9 \mathrm{~Kb}$ was identified and cloned in PCR II. O nly the intron-exon boundaries of an intron of approximately $9 \mathrm{~Kb}$ were sequenced as shown in Figure 1. This intron, located in the sequence coding for the cleaved signal peptide of $\mathrm{SCN} 2 \mathrm{~B}$, was not identified in previous work on the rat gene. ${ }^{7} \mathrm{U}$ sing primers designed on the intron-exon boundaries al ready established, we amplified the other two introns of 289 and $977 \mathrm{bp}$ from the same Y A C clone $665-\mathrm{E}-10$, thus confirming the results obtained starting from genomic DNA.

The established exon-intron structure of the SCN 2B gene is schematically illustrated in Figure 2. B ased on this gene structure, we searched for mutations in the SCN 2B genes of our CM T4B patient sample consisting of five affected individuals from the inbred Italian pedigree used to map the CM T 4B gene $(1945,273,279$, 287 , and 1943 from the pedigree reported in reference 4), two affected sibs born from unaffected parents and one sporadic CM T 4B case. ${ }^{12}$ We amplified the 4 exons of the SCN2B gene using primers designed for the intron boundaries. O nly the wild-type allele was found in all patients by analysis of the entire coding sequence of each gene.

A t the same time, we performed a refined physical localisation of the $S C N 2 B$ gene to confirm its previous location by FISH on chromosome 1lq23. A search in the CEPH YAC contig map of the human genome ${ }^{13}$ allowed us to assign the positive clones, retrieved through PCR screening of the CEPH YAC library, to two clusters of clones recognised by $G$ énéthon's markers D 11S1340, D 11S939, and D 11S1356. These markers are located $20 \mathrm{~cm}$ distal to the critical interval for

$\begin{array}{cccccccc}\text { 5' UTR } & \text { EX 1 } & \text { INT 1 } & \text { EX 2 } & \text { INT 2 } & \text { EX 3 } & \text { INT 3 } & \text { EX 4 } \\ 190 \mathrm{bp} & 70 \mathrm{bp} & 9 \mathrm{~Kb} & 168 \mathrm{bp} & 298 \mathrm{bp} & 217 \mathrm{bp} & 977 \mathrm{bp} & 200 \mathrm{bp}\end{array}$

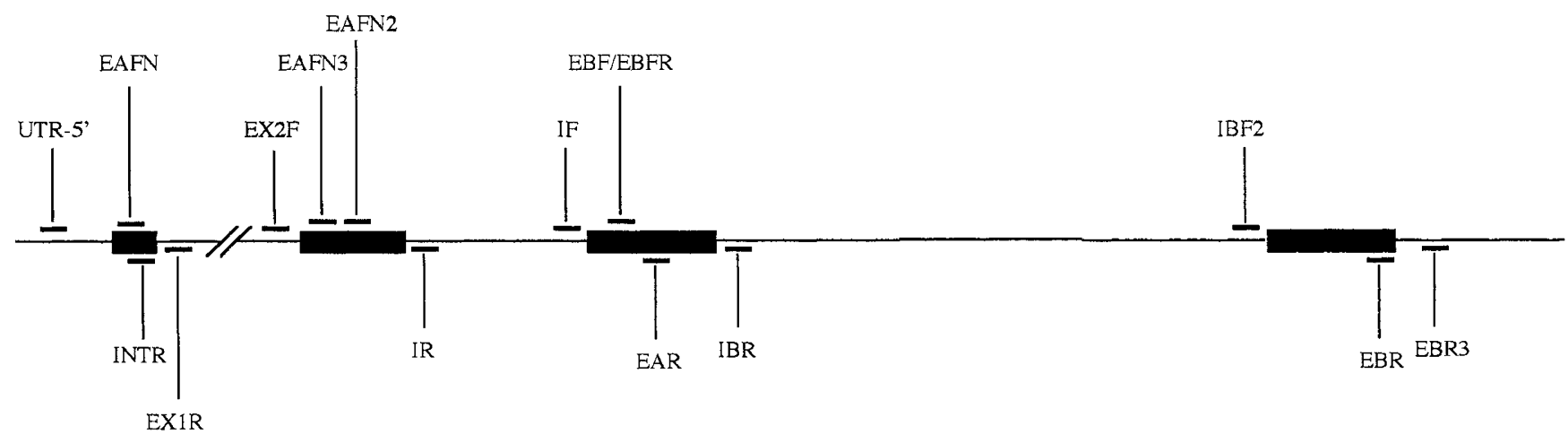

Figure 2 The intron-exon organisation of the SCN2B gene is schematically reported. The size of each intron or exon is also indicated. 
CM T4B, which is delimited by markers D $11 S 1332$ and D 115917 located in 11q22.1, as determined from the same YAC contig map. To confirm these results which excluded the $S C N 2 B$ gene from the region of interest, we screened by PCR a panel of radiation hybrids using $E B F / E B R$ as primers (Figure 1 and Table 1 ). Thus, the SCN 2B gene was mapped on chromosome 11q23 at 1.92 CR from the ST S marker W I-7642 of the integrated map from the Whitehead Institute (http://www-genome.wi.mit.edu/). This STS is flanked by G énéthon's markers D 11S1340 proximally and D11S936 distally, which are located $20 \mathrm{CM}$ distal to the lower limit of the $C M T 4 B$ region. We therefore concluded that $S C N 2 B$ is not the CMT4B gene. To our knowledge, no other disease has been mapped to the $11 q 23$ region for which SCN2B might constitute a suitable candidate gene.

\section{Acknowledgements}

We thank D r C inzia Sala and D r M ita M ancini from the Y A C screening Centre DIBIT, M ilan, I taly, and M r Loris Bernard and Dr Sandro B anfi from TIGEM, DIBIT, M ilan, Italy for the $\mathrm{R}$ adiation $\mathrm{H}$ ybrids mapping. This project is supported by Telethon-I taly, grant no. E 598 (M D) and NIH grant NS25704 (WAC).

\section{References}

1 Ben $O$ thmane $K$, H entati $F$, L ennon $F$ et al: Linkage of a locus (CM T4A) for autosomal recessive Charcot-M arieTooth disease to chromosome 8q. H um M ol G enet 1993; 2: 1625-1628.

2 O hnishi A, Muray $Y$, Ikeda M, Furuya $H$, Kuroiwa $Y$ : A utosomal recessive motor and sensory neuropathy with excessive myelin outfolding. Muscle Nerve 1989; 12: 568-578.
3 Q uattrone A, Gambardella A, Bono F et al: A utosomal recessive hereditary motor and sensory neuropathy with focally folded myelin sheaths. Clinical, electrophysiologic and genetic aspects of a large family. N eurology 1996; 46: 1318-1324.

4 Bolino A, Brancolini $\mathrm{V}, \mathrm{B}$ ono $\mathrm{F}$ et al: Localization of a gene responsible for autosomal recessive demyelinating neuropathy with focally folded myelin sheaths to chromosome 11q23 by homozygosity mapping and haplotype sharing. $\mathrm{H}$ um M ol G enet 1996; 5: 1051-1054.

5 Eubanks J, Srinivasan J, Dinulos MB, Disteche CM, Catterall WA : Structure and chromosomal localization of the $\beta 2$ subunit of the human brain sodium channel. NeuroR eport 1997; 8: 2775-2779.

6 Isom LL, De Jongh KS, Catterall WA : A uxiliary subunits of voltage-gated ion channels. Neuron 1994; 12: 1183-1194.

7 I som L L, R agsdale D S, De Jongh K S et al: Structure and function of the $\beta 2$ subunit of brain sodium channels, a transmembrane glycoprotein with a CAM motif. Cell 1995; 83: 433-442.

8 De Jonghe P, Timmerman V, Nelis E, Martin JJ, Van B roeckhoven $C$ : Charcot- $M$ arie-Tooth disease and related peripheral neuropathies. Journal of Peripheral Nervous System 1997; 2: 370-387.

9 Sambrook J, Fritsch E F, M aniatis T: M olecular Cloning. A $\mathrm{L}$ aboratory $\mathrm{M}$ anual. Col Spring $\mathrm{H}$ arbor $\mathrm{L}$ aboratory Press, 1989.

10 Green ED, O Ison MV: Systematic screening of yeast artificial-chromosome libraries by use of the polymerase chain reaction. Proc Natl Acad Sci USA 1990; 87: 1213-1217.

11 Walter MA, Spillett DJ, Thomas P, Weissenbach J, Goodfellow PN. A method for constructing radiation hybrid maps of whole genomes. Nat Genet 1994; 7: 22-28.

12 Schenone A, A bbruzzese M, U ccelli A et al: Hereditary motor and sensory neuropathy with myelin outfolding: clinical, genetic and neuropathologic study of three cases. J Neurol Sci 1994; 122: 20-27.

13 Chumakov IM, Rigault P, Le Gall I et al: A YA C contig map of the human genome. Nature 1995; 377 (suppl): 175-297. 